\section{ESTÉTICA FENOMENOLÓGICA. LA OBRA DE ARTE ARQUITECTÓNICA}

\author{
Pau Pedragosa \\ Departamento de Composición Arquitectónica \\ Escola Tècnica Superior d'Arquitectura de Barcelona \\ Universitat Politècnica de Catalunya
}

\section{PHENOMENOLOGICAL AESTHETIC: THE ARCHITECTONIC WORK OF ART}

\begin{abstract}
The purpose of this article is to discuss the possibility and task of aesthetics under the phenomenological point of view. I focus on architecture and analyse its stratified structure, according to Roman Ingarden's analysis, and the specific character of the aesthetic attitude against other attitudes that architecture also demands, according to the observations of Paul Valéry. I finally raise the central question about the possibility of the understanding art in general and architecture in particular, comparing phenomenological aesthetics and aesthetics of negativity.
\end{abstract}

KEY WORDS: Aesthetics, Phenomenology, Architecture, aesthetic experience, transcendence-inmanence, autonomy-heteronomy of art, aesthetics of negativity, E. Husserl, R. Ingarden, P. Valéry.

\section{INTRODUCCIÓN}

El título "estética fenomenológica" requiere una aclaración previa. La estética nace en el siglo XVIII como disciplina filosófica que se ocupa del ámbito del conocimiento sensible -inferior y confuso frente al conocimiento racional- cuyo objeto es la belleza. A finales del XVIII y principios del XIX el conocimiento sensible y la belleza se circunscriben en el campo del arte, de manera que la estética acota su ámbito a la belleza artística y se define como filosofía del arte. Establecido su campo, surge el problema de cómo proceder en su investigación. Debido a que la estética como disciplina filosófica se formó y consolidó durante el Idealismo alemán, la filosofía del arte y de la belleza fue el resultado de la "deducción" de un sistema filosófico preestablecido, de manera que sus principios se obtuvieron de una instancia ajena a la propia experiencia de las obras de arte. Con la deducción de una estética metafísica se sustituye la experiencia inmediata y concreta de las obras de arte, quedamos dispensados de ellas de manera que éstas pueden desaparecer o sólo servir de ejemplos. Devolver a la estética su concreción y recuperar la experiencia de las
RESUMEN: El propósito de este artículo es discutir la posibilidad y tarea de la estética bajo un enfoque fenomenológico. Nos centramos en la arquitectura, de la cual analizamos su estructura estratificada, siguiendo los análisis de Roman Ingarden, y la especificidad de la actitud estética, frente a otras actitudes que también reclama la arquitectura, según las observaciones de Paul Valéry. Planteamos finalmente la cuestión central acerca de la posibilidad de la comprensión del arte en general y de la arquitectura en particular comparando la estética fenomenológica con la estética de la negatividad.

PALABRAS CLAVE: Estética, fenomenologia, arquitectura, experiencia estética, trascendencia-inmanencia, autonomía-heteronomía del arte, negatividad estética, E. Husserl, R. Ingarden, P. Valéry.

obras de arte es el propósito de la estética fenomenológica. Con el adjetivo "fenomenológico" aplicado a la estética, se pone todo el énfasis precisamente en las obras de arte, éstas son las cosas mismas a las que hay que remitirse y de las que hay que extraer sus momentos esenciales a través de la instancia última que la fenomenología reivindica para obtener conocimientos con evidencia, la intuición. La intuición no es una facultad mágica capaz de ver las esencias de las cosas, de las obras de arte, en este caso, sino que es simplemente la conciencia del objeto en su presencia directa e inmediata, "en persona". Intuir una obra de arte, por ejemplo, una obra arquitectónica, significa ver el edificio, recorrerlo por dentro y por fuera, tocarlo, escucharlo, aprehenderlo en la integridad de la experiencia arquitectónica. Aunque la estética debe dar cuenta de los diferentes géneros artísticos, aquí nos vamos a centrar sólo en la arquitectura. En tanto que vamos a ensayar una estética fenomenológica no nos interesa dar una interpretación de una obra de arte arquitectónica individual, aunque a obras concretas hay que remitirse, sino comprender la esencia de la arquitectura en general, la estructura esencial de la obra de arte arquitectónica y de la experiencia estética en que 
la obra aparece. Para ello hay que poner todo el esfuerzo en ver pacientemente lo que efectivamente vemos cuando experimentamos y recorremos un edificio, pues es en esta percepción atenta y demorada, recuperada y repetida, donde podemos comprender la obra de arte arquitectónica.

En nuestra aproximación a la estética desde un punto de vista fenomenológico descubriremos la problemática sobre el estatuto de la obra de arte y de la experiencia estética. Esta problemática recorre la estética moderna del siglo XX y se manifiesta en dos tradiciones: la estética de raíz fenomenológica-hermenéutica y la estética de la negatividad. La primera ve en la experiencia del arte uno de los diferentes modos de experiencia, un modo que, aunque privilegiado en ciertos aspectos con respecto a los otros, no compite con ellos; mientras que la segunda le atribuye un potencial subversivo, negativo, respecto a los otros modos de experiencia. La estética negativa está determinada por el concepto de autonomía, la experiencia del arte es independiente de cualquier otra experiencia y su sentido (si es que hay alguno) no puede traducirse o equiparase a sentidos no estéticos; la estética de cuño fenomenológico define la experiencia estética como heterónoma pues el sentido de la experiencia depende de experiencias adquiridas fuera del campo del arte. En nuestro intento de describir la estética desde un punto de vista fenomenológico, explicaremos la seria objeción que le plantea la estética de la negatividad.

Este escrito es sobre estética y no sobre poética; la primera se refiere a la experiencia que un observador tiene de las obras de arte acabadas para comprender la estructura de esta experiencia y la de su objeto, la segunda tiene por tema el proceso creativo del autor de la obra. Aquí nos vamos a centrar sólo en la experiencia que un observador tiene de la obra arquitectónica acabada.

\section{ANÁlisIS FENOMENOLÓgICO DE LA PERCEPCIÓN ${ }^{1}$}

El análisis fenomenológico muestra que la percepción de cualquier objeto material en el espacio, como un edificio, es siempre parcial, incompleta, no ve nunca todo el edificio, sino sólo alguna de sus partes, un lado o una cara del edificio, dejando fuera de la vista otros lados que aunque no estén presentes hay la conciencia de que se podrían ver si se diera la vuelta al edificio o se entrara en su interior. Husserl denomina "lados" o "caras" (Seiten) a las partes percibidas del objeto, a las vistas, las perspectivas, las parcialidades que lo hacen presente a la percepción. No hay que concluir que porque se perciban directamente, físicamente ahi delante, "en persona", sólo partes incompletas de todo el edificio, no se lo perciba de hecho todo entero pues, obviamente, se está enfrente de él y se percibe todo el edificio desde el comienzo, pero lo que ocurre es que el edificio "entero" visto, por así decir, como "de golpe" no se presenta con la evidencia intuitiva con la que vemos uno de sus lados o partes, pues hay lados que están ocultos o se ven de manera borrosa respecto a la posición desde la que miro; el edificio como un todo, del que tengo una serie de "intenciones llenas" e "intenciones vacias" "sólo" con una presencia vaga e imprecisa, aunque suficiente para que se lo comprenda y uno pueda ocuparse de él de algún modo, ya sea para usarlo según su función, para seguir observándolo o cualquier otra actividad asociada al edificio en cuestión. Es importante destacar que la totalidad del objeto dada ya de antemano, precisamente por ser una donación vacía, espera ser "llenada" y de este modo estipula las maneras posibles de acabar de percibirlo, de "llenar" la intuición primera, marcando las direcciones que hay que seguir, que hay que realizar para completar la percepción del objeto, para ver con evidencia intuitiva el objeto que sólo de una manera vacía y vaga se ha presentado al principio a la percepción ${ }^{3}$.

El análisis muestra todavía que esta relación entre el objeto y los lados en que se presenta, se da también entre estos mismos lados y los escorzos parciales en que se dan ${ }^{4}$. El lado que se ofrece directamente a la visión, la fachada norte de un edificio por ejemplo, tampoco se percibe de golpe toda entera, sino sólo en una vista determinada, aparece en distintas formas trapezoidales, debido al escorzo de la perspectiva, hasta convertirse en una línea a medida que se desplaza el ángulo de visión. Así pues, los lados o partes del edificio se presentan en y a través de muchas otras partes, "aspectos", "perfiles", "escorzos", "matices", términos todos estos que recogen el hecho esencial a toda percepción según el cual la identidad de un objeto o parte de éste sólo se da en manifestaciones parciales siempre cambiantes 0 , dicho al revés, un objeto o parte de éste nunca aparece de manera completa y definitiva, no hay nunca una donación plena y acabada que agote la identidad del objeto, siempre habrá otra posible donación de lo mismo bajo otro punto 
de vista, con tal o cual reflejo de la luz que desvela un aspecto nuevo, una cualidad antes desapercibida ofrecida desde tal distancia, otra manera de agrupar y componer la secuencia de partes del edificio, y todo ello incluyendo, además, los cambiantes estados subjetivo-psicológicos del observador. El hecho de que no hay nunca, por principio, una percepción totalmente acabada del objeto, que sea la definitiva, es lo que hace a la percepción esencialmente inadecuada ${ }^{5}$.

Husserl utiliza el término, difícil de traducir, de Abschattungen, para expresar toda esa multiplicidad de partes perceptivas del objeto. El término alemán abschatten significa "esbozar", "perfilar", "proyectar", "hacer un esquema" de algo, en el sentido en que un arquitecto esboza y dibuja ${ }^{6}$. En este sentido los escorzos y perfiles son como los esbozos del arquitecto que proyecta la forma definitiva del edificio, sabiendo, sin embargo, que dicha forma no se da más que en estos esbozos, que no hay forma definitiva si no está esbozada de algún modo, aunque tampoco los dibujos darán definitivamente toda la forma. De manera parecida, en la percepción que un observador tiene del edifico, su tamaño, su forma, su posición, las relaciones entre sus partes y con otros objetos del entorno, "se dibujan" de distintos modos según el movimiento y cambio de perspectiva y no hay otra manera de percibir todo el edifico que no sea en y a través de estos "dibujos". Una Abschattung es el esbozo o dibujo de un aspecto de un objeto, pero que incluye la ambigüedad de ser tanto el proceso subjetivo de esbozar y dibujar (un episodio de nuestra sensibilidad) como la forma esbozada (presenta la cualidad del objeto). La ambigüedad de la Abschattung consiste en que expresa tanto las impresiones subjetivas como las presentaciones objetivas, tanto los episodios de mi sensibilidad, como la presencia sensible de los objetos; es a la vez una parte del objeto que está siendo percibido y los estados de la sensibilidad que están percibiendo. Como partes de la sensibilidad, los perfiles (Abschattungen) también pueden llamarse "impresiones"7. Hay una correlación estricta entre "lo percibido", el objeto, el lado, y "la percepción" sobre ello, el perfil, el aspecto, el escorzo, la impresión, es decir, entre la identidad del objeto y la donación o presentación de la identidad.

Si ponemos ahora la atención, no en el aspecto objetivo de los perfiles que muestran el objeto, sino en el subjetivo, entonces nos hacemos conscientes de la sensibilidad y del cuerpo, donde ella se "ubica". Como episodios de mi cuerpo vivo, los perfiles, las impresiones, muestran la espacialidad viva de mi cuerpo: El cuerpo vivo se constituye como el campo donde los episodios sensibles tienen lugar, lugar que tiene un tipo apropiado de localización y movilidad que no coincide con la localización mundana de las formas y los movimientos de las cosas, pues es la condición de posibilidad de la espacialidad mundana. El movimiento del cuerpo, que transforma continuamente los aspectos de los objetos espaciales, es un movimiento vivido internamente en la inmanencia del cuerpo sentido y es este movimiento vivido el que imprime dinamismo al proceso continuo de la percepción; este movimiento inmanente está ligado a los ejes de orientación del cuerpo, cuyo centro absoluto, el aquí, es siempre y constantemente este cuerpo propio que sube y baja, va hacia delante y hacia atrás, a la derecha y a la izquierda, se acerca y se aleja y, por tanto, no es percibido en los términos en que estamos describiendo la percepción de objetos en el espacio. El campo de la espacialidad interna (o protoespacialidad) y las capacidades cinestésicas de mi cuerpo vivo definen y redefinen a cada paso el centro-aquí de orientación, lo desplazan consigo y, con él, cambian continuamente la ubicación de los objetos entre sí y la de éstos en relación con el cuerpo ${ }^{8}$.

Llegados a este punto nos preguntamos qué tipo de relación hay entre el edificio, ese objeto en el espacio que estamos percibiendo, y toda esta pluralidad de perfiles en que el edificio se hace presente a la sensibilidad. Toda esta pluralidad continuamente cambiante se refiere a un mismo objeto, el edificio, que no cambia en su identidad como tal edificio. Todos los perfiles, por muy diferentes que sean entre sí, tienen en común el hecho de que los percibo como pertenecientes a uno y el mismo objeto, a este edificio ahí enfrente. La multiplicidad se da sobre una identidad, la del objeto, que está ahí y se muestra continuamente mientras lo estoy percibiendo. Sin embargo, hay que notar que la identidad del objeto, la objetividad hacia la que convergen sintéticamente los múltiples aspectos, no es ni un aspecto más ni la suma de todos ellos, sino que la identidad del objeto pertenece a un orden diferente del de los aspectos sensibles. La identidad no aparece como un aspecto, cara o perfil, sino como la unidad de esta corriente de perfiles. Este tipo de relación es, según la Fenomenología, de trascendencia ${ }^{9}$; el objeto trasciende sus presentaciones, y esto significa que el objeto es más que sus presentaciones sensibles, puede darse una y otra vez en otras apariencias. Y, a la vez, dicho por el otro lado, los perfiles del objeto nunca

(1)


agotan la identidad del objeto. Cuando somos conscientes de que las impresiones actuales no ofrecen más que una pequeña parte de todo lo que podemos percibir del objeto, entonces somos conscientes de la trascendencia del objeto. Que la identidad sea trascendente a la multiplicidad no quiere decir que sea separable; según Husserl la identidad se $f u n d a^{10}$ en esa pluralidad, es una parte no independiente, un momento, fundada en ella. No hay identidad sin síntesis, sólo hay este edificio en sus múltiples donaciones y en ningún otro sitio o de ninguna otra manera.

De esto se desprenden dos caracteristicas de la percepción" ${ }^{11}$ que son de la mayor importancia para comprender el fenómeno estético. Ambas se desprenden de la esencial inadecuación de la percepción de objetos en el espacio. La primera es que la identidad del objeto desborda todas las visiones parciales que de él se tienen; la segunda, paradójicamente inversa a la anterior, es que son estos aspectos parciales los que desbordan la identidad a la que se refieren. Si la identidad del objeto como un todo desborda siempre las visiones parciales que de él se tienen, no hay más salida que la continuada búsqueda de más vistas que enriquezcan lo ya visto con nuevos matices, que precisen lo que no se ve con claridad y que muestren lo todavía no visto $^{12}$. Esta continua búsqueda no es, sin embargo, una mera repetición de "lo mismo" pues toda revisión es una nueva versión, siempre diferente, de lo mismo ${ }^{13}$; siempre habrá otro matiz, otro escorzo, que enriquece la experiencia del edificio. La inadecuación de la percepción es lo que hace que el objeto sea como un punto de fuga en el infinito hacia el que convergen todas sus donaciones sensibles sin que ninguna de las secuencias de donaciones, ni siquiera la suma de todas ellas, lo expongan por completo; según esto, el objeto siempre es más que sus concreciones sensibles. La segunda caracteristica que hemos destacado pone el énfasis en que el objeto es menos que sus concreciones, la identidad del objeto trascendente parece demasiado poco frente a la infinita variedad y riqueza sensible de sus apariciones. Si la identidad es el polo hacia el que tiende la multiplicidad de presentaciones sin nunca alcanzarla, si la identidad del objeto sólo se presenta de modo incompleto, fragmentario, imperfecto, entonces sólo se puede compensar esta deficiencia de ser provisional con la obstinación testaruda de seguir persiguiendo la siempre escurridiza objetividad de lo que vemos, de seguir insistiendo, impulsados por el afán de máxima precisión inscrito en la búsqueda de la "cosa misma", en la proliferación de aspectos, en multiplicar las vistas que exponen cada lado, cada parte. Resultando de ello la conciencia de que la infinita proliferación de matices sigue siendo insuficiente, pero no por ser aún demasiado poco, por faltar más, sino por todo lo contrario, por ser demasiado, por el exceso desbordante de posibilidades de darse el objeto, pues a cada nueva vista siguen nuevas anticipaciones de otras posibles que, al actualizarse, piden constantes revisiones de lo visto previamente, sin poder superar su esencial inadecuación al objeto, el hecho de que no encajen una vistas con otras, no compongan la presentación definitiva, estable y coherente del objeto en cuestión. En este sentido la riqueza sensible supera la identidad del objeto, lo "empequeñece", sin dejar nunca de referirse a él como el polo al que tiende. Si de la primera caracteristica resulta un empobrecimiento e imperfección de lo sensible, de la segunda el empobrecimiento cae del lado de la identidad del objeto en comparación con su exuberante exhibición. De ambas circunstancias obtenemos dos resultados esenciales para comprender la obra de arte: La trascendencia siempre desbordante del objeto es característica de la obra de arte y la proliferación tendencialmente infinita de aspectos sensibles constituye la experiencia estética, caracteriza el ámbito de lo estético: la sensibilidad. Como veremos más adelante, ambas caracteristicas se resumen con el término "esquema" mediante el cual Roman Ingarden recoge la característica esencial de la obra de arte y la experiencia estética.

\section{LA ACTITUD FENOMENOLÓGICA Y LA TAREA DE LA ESTÉTICA FENOMENOLÓGICA}

Antes de tomar en consideración la arquitectura como arte hay que volver la mirada hacia atrás y ver cómo se ha hecho esta descripción de la percepción que hemos llevado a cabo hasta aquí. Esta es una descripción fenomenológica en la cual nos hemos hecho conscientes de la diferencia entre el objeto y la pluralidad de sus manifestaciones. Sólo una vez que hemos tomado el giro hacia la actitud fenomenológica nos hemos hecho conscientes de los objetos con los que nos ocupamos cotidianamente como identidades en una multiplicidad de perfiles; sólo entonces los perfiles se hacen temáticos. Este interés por las apariencias de los objetos más que por los objetos mismos es lo que define la actitud fenomenológica. De ahí su nombre: "ciencia de los fenómenos", de la cosas en 
su aparecer, en tanto que fenómenos. En la actitud natural -expresión que usa la fenomenología para referirse a nuestro vivir cotidiano- estamos inmersos en los objetos y vivimos, experimentamos, sus apariencias sólo "a través", sin llegar a verlas, como quien mira a través del cristal de unas gafas sin ver el cristal mismo que le permite ver. En la actitud natural damos por supuesto que nos ocupamos con objetos idénticos, que mi casa siempre sigue siendo el mismo edificio al que entro, habito y del que salgo, pero no apreciamos las diferencias, los diferentes aspectos dentro de los cuales esa identidad se mantiene. Cuando entramos en la actitud fenomenológica, los diferentes perfiles que eran experimentados pero no tematizados, mirados pero no vistos, entran en el foco de nuestra atención y aparece la diferencia entre la multiplicidad de apariencias y el objeto, nos interesamos no por el objeto, sino por el objeto en su aparecer, por el darse del objeto, y comprendemos la trascendencia del objeto respecto a sus modos de darse ${ }^{14}$.

La tarea de la fenomenología consiste entonces en describir qué tipo de apariencias están involucradas en hacer concreta e intuitiva la presencia del objeto en cuestión. Los errores más frecuentes ocurren cuando se confunden los aspectos correspondientes a una objetividad con los de otra, porque entonces se yerra en hacer intuitiva y concreta la presencia del objeto en cuestión. Aplicada a la estética, la tarea de la fenomenología consiste en describir las múltiples apariencias que corresponden a una obra de arte, en nuestro caso, a la obra de arte arquitectónica, sin confundirlas con las que corresponden a un objeto material en el espacio -a un edificio-, pero sin confundirlas tampoco con las que corresponden al edificio entendido como objeto cultural -una iglesia, un teatro-. El análisis fenomenológico permite deshacer la equivocidad del término "arquitectura" que significa tanto un edificio como objeto material, una obra arquitectónica como objeto cultural y una obra de arte arquitectónica como objeto estético. Roman Ingarden, siguiendo a su maestro Husserl, llama "estratos"15 (Schichten) a los dos momentos constitutivos de toda objetividad (la identidad y la multiplicidad en que ésta se muestra). Para percibir el tipo de multiplicidades apropiadas a cada objetividad se debe tomar una actitud u otra.

Un primer nivel en que la identidad de un objeto material se presenta son los cambios potencialmente infinitos a que está sujeto según lo que pasa a su alrededor. Forma parte del sentido esencial de una cosa material su interacción causal con otras cosas y con el entorno; la cosa material está regida por ciertas leyes causales que permiten hacer predicciones posibles de su comportamiento: "Este todo [el edificio] posee exclusivamente propiedades físicas, por ejemplo, cierta extensión y posición en el espacio real que bajo la influencia de factores externos cambia dentro de ciertos limites determinados por el tipo de materiales del que está hecho. Cuando la temperatura cae, se contrae; cuando la temperatura asciende, se expande [...] No se debería ni llamar a este todo real un 'edificio.' Pues un 'edificio' es un objeto cultural ónticamente dependiente de nuestras actitudes. [Sin embargo] para abreviar, lo llamamos 'edifi-


plicidad de aspectos en que se presenta, es la base para otras objetividades que podemos constituir dependiendo de la actitud que tomemos respecto a él. Con el cambio de una actitud a otra no se anula la identidad de la cosa material con respecto a la cual nos comportamos de una manera o de otra, pues cada nueva actitud despliega toda una serie de aspectos múltiples que confieren al edificio nuevas características que se dan como momentos que ocurren en la objetividad en cuestión y llevan a la constitución de una nueva objetividad: "Para que algo como una 'iglesia', un 'teatro' o un 'parlamento' se origine de un todo consistente en la disposición de cierta cantidad de materiales de construcción (piedra, hierro, ladrillo, etc.) se requiere una actitud subjetiva completamente diferente por parte de la gente que se ocupa con tales objetividades, así como la realización de toda una serie de actos conscientes de un tipo especial"17. Así, sobre la base de un edificio como objeto material constituimos objetos culturales como una iglesia, un teatro o un parlamento y sobre éstos se puede tomar otra actitud, la estética, que constituye una obra de arte como objeto estético: "Para aprehender una obra arquitectónica, en la ocupación inmediata con ella, como obra de arte, y en particular como objeto estético, se debe adoptar una actitud especial hacia el edificio que difiere esencialmente tanto de la puramente cognoscitiva y de la religiosa, como de la del hombre práctico. Esta actitud especial tiene como correlato intencional un nuevo objeto" ${ }^{18}$.

La actitud que constituye el edificio como obra de arte es la percepción o experiencia estética, la cual no descubre propiedades físicas ni culturales, sino cualidades estéti$\operatorname{cas}^{19}$. Tan pronto como descubrimos que un edificio es una obra de arte nos damos cuenta de que el edificio ya no lo 
tomamos meramente como un objeto material, sino como algo que de alguna manera va más allá de la materialidad del edificio, hasta el punto de que esta materialidad, entendida como interacción causal con el entorno, no juega ningún papel en nuestra consideración del edificio como obra de arte. Tampoco tomamos en consideración sus rasgos culturales, los que, por ejemplo, tenemos en cuenta cuando, como espectadores, asistimos a un teatro. A la multiplicidad correspondiente a la identidad de la obra de arte, bien distinguida de la que constituye un edificio o un objeto cultural, Ingarden la Ilama multiplicidad de objetos estéticos.

\section{LA OBRA DE ARTE AROUITECTÓNICA COMO FORMACIÓN ESQUEMÁTICA}

Podemos caracterizar la experiencia estética del siguiente modo: Consiste, por un lado, en mantener bien distinguidos los dos estratos que articulan la obra de arte - la identidad de la obra y las múltiples maneras cómo esta identidad se muestra de manera concreta- y, por el otro lado, en poner toda la atención en el segundo estrato, descrito por Ingarden como "una multiplicidad de esquemas de aspectos a través de los cuales la forma espacial se hace presente cuando un espectador, en su experiencia consciente, actualiza esos esquemas $y$, asi, los concretiza"20.

Con el término "esquema" Ingarden se refiere a las Abschattungen de Husserl, a los sucesivos perfiles que se van actualizando, concretizando, para constituir la identidad de la obra de arte. A cada actualización de estos esquemas, que dependen de los diferentes observadores e, incluso, a las diferentes concreciones de un mismo observador en diferentes tiempos, corresponde un objeto estético: "Ante todo hay que notar que tan pronto como una obra de arte arquitectónica está incorporada en un edificio real [...] es un sólo objeto individual de un tipo específico y con un específico modo de ser. En contraste, puede haber toda una multiplicidad de objetos estéticos arquitectónicos que se forman sobre la base de una y la misma obra de arte arquitectónica, por diferentes observadores que realizan una experiencia estética"21.

Detengámonos en los esquemas, el rasgo definitorio de toda obra de arte, no sólo de la arquitectónica, y lo que permite precisar con más detalle la estructura de la obra de arte y de la experiencia estética. Lo que está en cuestión con este término son los perfiles o aspectos de un objeto pero no tal como los experimentamos en su concreción sólo una vez en la percepción actual, sino más bien ciertas idealizaciones que forman un esqueleto, un esquema, de todos los aspectos concretos, transitorios y movedizos. Un esquema hace referencia a una cierta regla de repetibilidad de los aspectos, a "una afiliación regular de una multiplicidad de aspectos"22. Hay una infinidad de aspectos correspondientes a un objeto pero todos ellos tienen en común que son partes del mismo objeto, no cualquier aspecto corresponde o hace concreto el objeto en cuestión, sino que éste determina qué aspectos le son propios y cuáles no y hay, por tanto, una regla de esa objetividad y es esta regla la que permite que los diferentes aspectos, por muy cambiantes que sean, se puedan repetir en tiempos diferentes. De esta manera los aspectos no percibidos de manera intuitiva no son solamente aspectos ausentes o "vacios", sino aspectos variables y prefigurados por esa regla que llamamos esquema. Los esquemas son esa estructura o esqueleto de los aspectos posibles que pueden concretizarse para que expongan un objeto. Estos esquemas no son, pues, otra cosa que aspectos esquematizados, "dejados listos", preparados $^{23}$, para su ulterior concreción en la experiencia actual. El término "esquema" da cuenta del hecho de que la experiencia de un objeto no es nunca ni tan abierta que no indique nada del curso ulterior de la percepción, ni tan cerrada que no se pueda nunca corregir. La estructura de la obra de arte está formada por dos estratos o dos niveles: el primero es la obra de arte misma entendida no como un objeto acabado y definitivo, sino como una formación esquemática susceptible de llenarse de infinitas maneras -aunque no de cualquier manera, sino sólo con las estipuladas por el esquema-; el segundo consiste en la multiplicidad de concreciones y actualizaciones de esos esquemas que se cumplen en cada experiencia estética de la obra. Cada una de esas concreciones es un objeto estético entendido como el correlato intencional de una multiplicidad coherente de percepciones parciales del edificio realizadas en la experiencia estética. Los objetos estéticos, resultado de la concreción de los esquemas, correlatos de las secuencias perceptivas tendencialmente infinitas, son siempre provisionales, cierran en una percepción estética lo que es esencialmente abierto a múltiples revisiones y actualizaciones: la obra de arte como esquema. Los infinitos objetos estéticos son la multiplicidad que tienden 
a esa objetividad siempre escurridiza que llamamos obra de arte, ésta es el polo al que tienden los desarrollos infinitos de la sensibilidad del observador. Por tanto, la obra de arte es una sola, tiene una identidad (aunque abierta, esquemática) mientras que hay infinidad de objetos estéticos correspondientes a una y la misma obra de arte. Con el término "esquema" o "aspectos esquematizados" se recoge el doble carácter de la percepción inadecuada que hemos establecido más arriba. El esquema pone el acento en la trascendencia de la obra de arte, en el hecho de su inalcanzable identidad, su carácter de fuente inagotable de múltiples apariciones, exhibiciones, revisiones e interpretaciones. Las infinitas concreciones del esquema ponen el acento en la multiplicidad de objetos estéticos que desbordan la identidad y definición de la obra de arte que parece empobrecida frente a la riqueza sensible de los objetos estéticos concretos. La obra de arte es a la vez mucho más, pues ningún objeto estético la agota, y mucho menos, pues la infinitud de objetos estéticos desborda y enriquece una obra de arte siempre más allá, inalcanzable e inaprensible en su totalidad.

Sin embrago, en el esquema resuena la noción de horizonte de Husserl: "Una cosa se da necesariamente en meros 'modos de aparecer', en que necesariamente hay un núcleo de algo 'realmente exhibido', rodeado, por obra de apercepciones, de un horizonte de algo 'co-dado' impropiamente y más o menos vagamente indeterminado. $Y$ el sentido de esta indeterminación está trazado de antemano, una vez más, por el sentido de lo percibido en cuanto tal y en general, o por la esencia universal de este tipo de percepción que Ilamamos percepción de cosas. La indeterminación significa, en efecto, necesariamente la posibilidad de determinarse en un estilo rigurosamente prescrito" 24 .

Ingarden reproduce en la experiencia de la obra de arte lo que es esencial a cualquier "percepción de cosas". ¿Qué es lo que distingue la percepción estética de cualquier otra percepción? ¿Qué diferencia hay entre una obra de arte y un objeto material?

\section{LA PERCEPCIÓN ESTÉTICA DE LA ARQUITECTURA}

Paul Valéry, que sabía ver muy bien la arquitectura, describe la problemática inherente a la percepción estética:
"La mayoría de la gente ve con el intelecto mucho más a menudo que con los ojos. En lugar de espacios coloreados, conocen conceptos. Una forma cúbica, blanquecina, alta y horadada por reflejos de cristal es para ellos, inmediatamente, una casa: ¡la casa! Idea compleja, concordancia de cualidades abstractas. Si cambian de lugar, el movimiento de las hileras de ventanas, la traslación de superficies que desfigura continuamente su sensación, se les escapa..., pues el concepto no cambia. Perciben, más bien, según un léxico que, según su retina, se aproxima tan mal a los objetos, conoce tan vagamente los placeres y padeceres de ver que han inventado las bellas perspectivas [...] Y todas las modulaciones que provocan los pequeños pasos, la luz, el entorpecimiento de la mirada, no les hacen mella. Ni hacen ni deshacen nada en sus sensaciones [...] iY, como desdeñan lo que carece de nombre, el número de sus impresiones se encuentra estrictamente limitado de antemano!"25.

Con "la mayoría de la gente" se refiere Valéry a la actitud natural de la vida cotidiana, actitud caracterizada como aquella que "ve con el intelecto mucho más a menudo que con los ojos", un ver que Le Corbusier atribuia a des yeux qui ne voient pas, y que consiste en ir directamente al objeto según un "léxico" grabado en la retina que identifica directamente el nombre del objeto; esta identificación procede de la manera siguiente: De entre las múltiples impresiones visuales o perfiles de los objetos, la mirada aísla sólo una cuantas que sabe interpretar, bien reconocibles por nosotros, y que le sirven de referencia para identificar los objetos. Esta limitación de las impresiones está determinada de antemano porque la identificación inmediata del objeto se da por el criterio del hábito, el cual introduce juicios elaborados y formados en nosotros desde la infancia, los cuales nos imponen continuidades, asociaciones. La costumbre impone una construcción de la visión que nos dispensa de ver mediante abreviaciones y sustituciones inmediatas que la primera educación nos ha enseñado. De las innumerables apariencias, impresiones, que nos asedian a cada instante, sólo una parte débil y muy pequeña es utilizable por nuestro vivir diario. Percibimos de los objetos sólo lo necesario para sustituirlo por una acción inmediata y determinada. La mayoría de nuestras percepciones o bien no sirven de nada y entonces no les damos ningún curso, o bien son puramente transitivas, nos sirven para algo y se cambian de inmediato por un reconocimiento de la identidad del objeto que nos mueve a alguna acción con él. No perdemos tiempo en la percepción porque ésta se 
resuelve en algo diferente de ella. Veo, por ejemplo, unas masas plásticas, unas superficies con huecos, entrantes y salientes, pero sus cualidades sensibles resultan abolidas y son sustituidas por la representación de una fachada y una hilera de ventanas, en definitiva, por la representación de una casa y por el comportamiento habitual asociado a ella. Éste es el caso de la actitud natural $y_{1}$ aunque más elaborada, también de la cognoscitiva, por ejemplo la del técnico arquitecto o ingeniero que ante la casa procede a rehabilitarla; todo lo que él percibe de la casa está completamente codificado por sus conocimientos, los cuales guían la selección de las impresiones relevantes y su adecuada interpretación. En estos casos lo sensible de los objetos no se conserva, no sobrevive a su comprensión, ha hecho ya su papel, "ha actuado; ha cumplido su función; ha hecho comprender: ha vivido"26.

Por el contrario, la arquitectura no muere por haber vivido ${ }^{27}$. Hay ocasiones en que las formas sensibles que percibimos del edificio adquieren tal importancia que se imponen y se hacen respetar $y$, también, desear $y$, por tanto, recuperar, repetir, sin abolirse en una representación y acto determinados 0 , dicho con más precisión, cualquier representación o reconocimiento del objeto al que inevitablemente se llega, es siempre provisional y reenvía a la inspección sensible. Cuando esto ocurre estamos delante de una obra de arte arquitectónica. El arquitecto busca el estado original de coordinación entre el ojo y los objetos, original por su carácter de principio, resultado de poner en crisis nuestras operaciones de identificación de los objetos, de reconocimiento habituales $y$, por tanto, de proponer al observador el reto de recrear lo que ve, como si lo viera por primera vez. Al observador la educación visual se le muestra insuficiente y entra en una actitud que le abandona al placer de instantes sin duración, de percepciones $\sin$ futuro $^{28}$ pues no se resuelven en nada más que en sí mismas. Ésta es la actitud estética, por su atención a la aisthesis, a la sensación y es la que la arquitectura, entendida como obra de arte, reclama y motiva al espectador para ejercerla. La percepción estética tiene su condición de posibilidad en la esencia de la percepción que hemos caracterizado como inadecuada. Por esta inadecuación la percepción está marcada por la búsqueda continuada de lo que la percepción muestra del objeto percibido, por la inspección de los múltiples aspectos, pues siempre habrá otro escorzo, otro matiz, que enriquece la experiencia del edificio.
Un edificio es un objeto espacial de tres dimensiones con una serie de espacios vacíos en su interior y puede, por tanto, verse desde fuera y desde dentro en una sucesión de diferentes lados y aspectos. Normalmente nos movemos alrededor y por el interior del edificio impulsados por intereses prácticos y actividades relacionadas con los quehaceres cotidianos; vivimos y nos movemos en función de lo que vemos pero no vemos más que lo que ocupa nuestro pensamiento y así no vemos nada que sea puramente visto. Las percepciones sólo las usamos en la medida necesaria para poder pasar a otra cosa. La selección muy limitada y guiada por el hábito de una serie de aspectos tiene por correlato la objetividad o parte de ésta que se llama edificio u obra arquitectónica como "teatro". Pero ciertos edificios o partes de éstos motivan o reclaman que se ejerza sobre ellos una mirada atenta y demorada. Adoptamos entonces la actitud estética cuyo correlato es una obra de arte arquitectónica. Esta detención y demora en los aspectos y perfiles es posible por los movimientos cinestésicos de los ojos que agudizan o desenfocan, que miran de soslayo y deslizan la mirada por las superficies, pero no como respuesta a objetivos prácticos, sino a las variaciones inherentes a los aspectos sensibles de los objetos, según el ritmo que impone el juego de los salientes y los entrantes, las concavidades y convexidades, los llenos y los vacíos; la mirada recorre la figura que trazan las nervaduras que articulan las superficies, detecta qué pausas le imponen, qué aceleración le imprimen, se pierde hacia el exterior a través de un hueco o se demora en la textura pesada de un muro y registra sus variaciones por medio del contacto corporal. El placer de lo sensible tiende a repetirse, reproducirse y recuperarse, aunque se puede agotar o interrumpir por algún factor externo, como la fatiga, que lo cancela y difiere su reinicio; la fatiga es una merma de la sensibilidad ${ }^{29}$ que nos hace descansar en el objeto, salir de la experiencia estética y volver a la actitud natural. La percepción estética es así fiel a la cosa misma de una manera que entra en íntima relación con la propia mirada fenomenológica. Es la cosa misma, los objetos percibidos 0 , con más precisión, los aspectos que percibimos de los objetos, las multiplicidades surgidas de nuestras percepciones y constituidas enteramente por relaciones internas y variaciones de la sensibilidad y no cualquier otro factor externo, los que determinan, a través de su hábil disposición por el arquitecto, las directrices que seguir para prolongar sus diferentes exhibiciones sensibles. Los aspectos sucesivos así dispuestos nos motivan a caminar 
en torno a las pantallas exteriores y a recorrer los espacios interiores, vista, tacto, oído y movimiento nos inducen a demorarnos en las impresiones, a conservarlas o renovarlas sin dar precipitadamente el salto a la identidad del objeto a la que las impresiones tienden.

La descripción de la percepción estética que hemos expuesto siguiendo a Valéry viene caracterizada por su oposición a la percepción no estética (natural, cotidiana). Si ésta consiste en una selección limitada de ciertos aspectos que alcanzan directamente su objeto, aquélla consiste en la imposibilidad de una selección de unos cuantos aspectos, en un proceso continuo de la percepción sin resultado final, por una oscilación entre el sonido y el sentido ${ }^{30}$, un balance perpetuo entre las partes sensibles (sonido) y el todo objetivo (sentido), que al no resolverse en una identidad tiene por resultado una inmersión en las percepciones y un aplazamiento de la comprensión de la objetividad hacia la que las percepciones remiten. Aquí surge la cuestión crucial para la estética sobre la posibilidad de la comprensión de la obra de arte. ¿Está la percepción estética enteramente circunscrita en el ámbito de las multiplicidades de aspectos sin poder trascenderlas hacia cierta objetividad que permita no sólo gozarlas, sino también comprenderlas? ¿Es lo esencial a la experiencia estética ser una multiplicidad pura, una inmanencia sin ninguna trascendencia de sentido? Según Ingarden, hay efectivamente objetos estéticos que son los correlatos intencionales de las multiplicidades sensibles: "Si de todas las percepciones parciales del edificio seleccionamos sólo aquellas que se realizan en la actitud estética [...] entonces pertenece a cada multiplicidad coherente de esos actos de aprehensión un correlato intencional que pertenece necesariamente a esa multiplicidad: un objeto estético" ${ }^{31}$.

Dentro de la experiencia estética hay un logro (Leistung) de las multiplicidades en una objetividad: Ésta resulta de la selección de una multiplicidad coherente de las percepciones realizadas en la actitud estética. Un objeto estético es la síntesis de una serie de multiplicidades estéticas y hay, por tanto, cierto acabamiento, síntesis, comprensión. El punto crucial de la problemática del sentido del arte gira en torno a la posibilidad de seleccionar sólo aquellas percepciones que se realizan en la actitud estética, dentro de la experiencia estética. A esta posibilidad se opone la estética de la negatividad.
La comprensión de la obra de arte. Autonomía o heteronomía de la obra de arte arquitectónica ${ }^{32}$.

Para concluir vamos a describir estas dos posiciones, la fenomenológica-hermenéutica y la negatividad. Obviando las muchas diferencias entre los autores, podemos incluir en la primera tradición a Ingarden, Gadamer $y_{\text {, en cierta }}$ manera también a Heidegger; en la segunda, también con ciertas simplificaciones, se agrupan los formalistas rusos, Adorno, Valéry (con muchos matices) y también Derrida (inscrito normalmente en la fenomenología). Ambas son excluyentes y definen dos de las posiciones más importantes en el campo de la estética moderna: Lo que está en debate es la definición del arte, la cual oscila entre la autonomía y la hetreonomía. El centro de la polémica consiste en la posibilidad de la comprensión del arte. La fenomenología afirma que hay una comprensión de un sentido estético en el arte, la negatividad lo niega o, más exactamente, dice que la comprensión estética consiste en el reiterado fracaso de llegar a alguna comprensión definitiva $y$, en la medida en que fracasa, revela el funcionamiento interno de la comprensión. Según la lógica negativa de la experiencia estética sólo quien aprehende las obras de arte en su relación negativa con todo lo que no es arte, puede comprenderlas en la lógica propia de su percepción ${ }^{33}$, que consiste precisamente en el diferimiento continuado de sentido o en el fracaso de toda tentativa de alcanzarlo. La cuestión crucial es: ¿Hay algún criterio interno a la secuencia de aspectos parciales que lleven a la síntesis de un todo de sentido estético comprensible? Veamos primero lo que tienen en común fenomenología y negatividad.

La fenomenología y la negatividad estética caracterizan la experiencia estética como la suspensión de toda decisión acerca de si los aspectos sensibles exhiben alguna objetividad. La experiencia estética, entonces, sólo puede consistir en la entrega a un deletreo paciente del proceso inmanente de la percepción de los aspectos ${ }^{34}$. Ahora bien, la procesualidad infinita de la sensibilidad abandonada al placer estético no es sólo una recepción pasiva de impresiones que ya están ordenadas, sino la actividad que las ordena $y$, así, el goce de la sensibilidad consiste en remontarse desde los sentidos ya constituidos en la experiencia natural al proceso de constitución de estos sentidos: "la mirada construye" 35 . Los sentidos, las objetividades, cristalizadas por el hábito y la costumbre, se

ARBOR CLXXXV 736 marzo-abril [2009] 355-367 ISSN: 0210-1963

(n)


disuelven en la experiencia estética y nos enfrentan a la tarea de constituirlos de nuevo, recrearlos, es decir, la experiencia estética nos traslada a la perspectiva interna de constitución, al proceso interno de la comprensión. En este sentido, la fenomenología contiene un primer momento de negatividad y de autonomía respecto a las experiencias no estéticas. La disputa entre la fenomenología y la negatividad radica en una diferente descripción de la relación entre la realización del proceso de constitución de objetividades y la objetividad constituida. Ambas afirman que el automatismo de la experiencia natural es ineficaz y es, así, cuestionado de raíz, pero mientras que la fenomenología dice que en el proceso de constitución de la experiencia estética se intensifica y enriquece la experiencia cotidiana, lo ya constituido fuera de la experiencia estética, la negatividad, en cambio, sostiene que el proceso de constitución no se resuelve en ningún sentido, se mantiene en el proceso sin salir de él, sin traer desde fuera ningún sentido y negando, así, el proceso de constitución. La fenomenología hermenéutica intensifica la experiencia cotidiana, la negatividad la subvierte ${ }^{36}$.

La experiencia estética, como hemos visto con Ingarden, consiste en actualizar los aspectos esquematizados en la obra de arte, estos esquemas son el horizonte de las posibles concreciones de los aspectos sensibles o cualidades estéticas de color, textura, luz, reflejos, sombreados, ritmos de las masas y los huecos, superficies escorzadas en movimiento, etc. Según el desarrollo hermenéutico de la fenomenología llevado a cabo por Gadamer, sobre el horizonte o esquema de todas las posibles perspectivas perceptivas, una experiencia estética particular abre una perspectiva que se despliega sobre el fondo de experiencias que el individuo ya ha realizado, pero con la peculiaridad de que estas experiencias se revelan ineficaces para comprender lo que se experimenta; se muestran pobres en comparación con la riqueza sensible que se despliega ante él. Por ejemplo, todos conocemos qué es una rampa o un pilar o una cúpula ¿0 mejor sería decir que sólo nos ocupamos con estos objetos pero en escasas ocasiones los vemos? ¿No queda demasiado "pequeña" o excesivamente pobre la identidad "rampa" para designar tanto lo que experimentamos en la Ville Savoye, como en el museo Guggenheim de F. LI. Wright? ¿Es eficaz llamar "pilar" al juego de reflejos adheridos sobre la superficie de los elementos verticales del Pabellón de Barcelona de Mies Van der Rohe? ¿No es extraño llamar "cúpula" tanto a la claridad estática y limpia de la cavidad que nos cubre en la capilla Pazzi de Brunelleschi, como al agitado claroscuro de cavidades y sólidos que acelera vertiginosamente la mirada en la iglesia de Sant'Ivo Della Sapienza de Borromini? Si son los movimientos de la mirada o, en general, las cinestesias del cuerpo, las que constituyen las objetividades, ¿constituimos la misma objetividad con cinestesias tan diferentes? Podemos decir que estas diferentes cúpulas son dos manifestaciones de una misma objetividad cultural, pero no el mismo objeto estético. Esa ineficacia o empobrecimiento de las experiencias propias ya conocidas (saber qué es una rampa, un pilar, una cúpula) que la experiencia estética revela, proyecta una mirada extraña sobre nuestra realidad conocida precisamente por ser demasiado conocida, demasiado obvia, tanto que ni la vemos. La experiencia estética implica la adquisición de una mirada extraña sobre lo propio, extrañeza necesaria para poder ver lo familiar como ajeno. La extrañeza característica de la experiencia estética no significa que el observador dirija su mirada a una realidad extraña, sino que dirige una mirada que le revela lo propio como extraño. No se trata de experimentar nuevos contenidos sino de ver lo ya conocido a una nueva luz que revela aspectos no vistos anteriormente; en la experiencia estética hay un re-conocimiento ${ }^{37}$. Por ser la experiencia cotidiana de una escalera demasiado obvia y trivial, hay que esperar a la experiencia estética de una escalera en una obra de arte arquitectónica para llegar realmente a verla y cuestionar nuestro conocimiento de tal objeto; la obra de arte nos abre los ojos. Si tenemos en cuenta que la arquitectura nos rodea siempre y en todos los lugares por los que nos movemos, que desde la cuna hasta la tumba -desde la casa hasta el monumento funerario- la arquitectura acompaña todos nuestros pasos, construye el escenario de nuestra existencia y da forma a nuestras costumbres, hábitos, acciones, movimientos y gestos en los que se resuelve nuestra existencia, entonces la experiencia estética de la arquitectura nos permite comprender de forma más rica e intensa estos aspectos esenciales de nuestra condición.

En la medida en que la experiencia estética logra una comprensión de las objetividades estéticas porque remiten a objetividades de la realidad extraestética, la experiencia estética es heterónoma y el sentido del arte depende de lo que no es arte. Esta remisión fuera de la experiencia significa que la inmanencia de la multiplicidad sensible se trasciende en una identidad de sentido, en una objetividad 
que ya no está en el plano de la pura sensibilidad. En cambio, según la negatividad estética, la experiencia estética es autónoma, independiente respecto a la experiencia no estética, el arte se define por su diferencia radical con respecto a lo que no es arte. En comparación con la heteronomía del arte, la autonomía está definida por la no trascendencia del sentido de la obra respecto a los aspectos que la exhiben. La experiencia estética es estrictamente inmanente y es incapaz de dar el salto trascendente a ningún sentido objetivo. La negatividad de la experiencia estética critica a la descripción fenomenológica haber tomado la decisión previa de que debe haber un sentido y así abandona el punto de visto interno del proceso infinito de la sensibilidad; no muestra si la inmersión en la experiencia estética produce por si misma algún sentido, sino que lo presupone. La negatividad estética se centra en la inmanencia de la experiencia estética y no ve en ella ningún logro de sentido pues no hay ningún criterio inherente al proceso infinito de la sensibilidad que lo estabilice en una identidad y critica a la fenomenología que lo tome de afuera. La negatividad pone de manifiesto el procedimiento fenomenológico: El criterio de la selección de los aspectos relevantes para lograr la síntesis de identificación y de acabamiento del proceso continuado de la experiencia estética sólo puede ser externo a la propia secuencia estética; es el criterio aprendido del hábito el que nos permite seleccionar aquellos aspectos correspondientes a un objeto $y$, por tanto, afirmar que sí hay un sentido, implica que se da una repetición, aunque transformada, de objetividades portadoras de sentido en el ámbito extraestético, que se repite un sentido ya conocido en la actitud natural. Desde la fenomenología se puede criticar a la negatividad diciendo que olvida que multiplicidad e identidad de sentido no son separables más que abstractamente, pues ambos son dos momentos que forman un todo concreto de sentido, un sentido susceptible de comprenderse de manera particularmente intensa y concreta en la experiencia del arte pero también, de alguna otra manera, en cualquier otro tipo de experiencia.

Tratándose de arquitectura, la heteronomía, la referencia a algo más allá de la obra de arte, parece además ser necesaria, pues a diferencia de las artes Ilamadas "libres" (pintura, música, poesía...), la obra de arte arquitectónica mantiene con su soporte, el edificio y el objeto cultural, una relación mucho más estrecha que, por ejemplo, la pintura con la tela como su soporte. Digamos que para la obra de arte arquitectónica es de la mayor importancia que su portador sea un "teatro" o un "museo", pues la multiplicidad de aspectos remiten a partes de esas objetividades como "platea" o "vestíbulo" y no se pueden disociar de ellas en vistas a la unidad coherente de la arquitectura; mientras que es relativamente menos importante para la pintura que su portador sea tela, madera o papel.

\section{NOTAS}

Recibido: 15 de noviembre de 2007 Aceptado: 15 de diciembre de 2007
1 Los análisis de Husserl de la percepción de un objeto material se hallan en muchas partes de su obra. Nos basamos aquí en los $\S \S 41-44$ de Husserliana III (Hua.III). Ideen zu einer reinen Phänomenologie und phänomenologischen Philosophie. Para una interpretación de estos análisis ver: Sokolowski, R., Husserlian Meditations. How Words Present Things, Northwestern University Press. Evanston, 1974, pp. 86-93.

2 Hua XIX/2. Logische Untersuchungen. Investigación VI. Los conceptos de intención vacía y llena se explican en los capítulos 1 y 2; el grado de completud, de "Ileno", en los 3 y 5. Las intenciones llenas y vacías son dos momentos de la conciencia de indentidad, correlativamente, la presencia y ausencia intuitiva del objeto son dos momentos del objeto.

3 Estas maneras regladas, estipuladas, definen la noción de "horizonte". La trataremos más adelante en relación con la percepción de la obra de arte.

4 Sokolowski, R., Ibid., p. 87.

5 Hua. III, § 44, p. 100.

6 Sokolowski, R., Ibid., p. 91.

7 ldem.

8 Husserl, E., Erfahrung und Urteil, Ed. L. Landgrebe, Hamburg: Claassen, 1954. 
$\S 19$. Desde el punto de vista de la espacialidad originaria se comprende la observación de Roman Ingarden a proposito de la arquitectura: "En cuanto al modo en que la obra arquitectónica nos es dada, no es de mucha importancia que normalmente seamos nosotros quienes nos movemos y no la obra, pues la obra aparentemente también se mueve en relación a nosotros". Ingarden, R., Ontology of the Work of Art, Ohio University Press, 1989, p. 305.

9 Hua III, § 41, p. 92.

10 Sobre la relación de fundamentación, Hua XIX/1. Logische Untersuchungen. Investigación III, § 14.

11 Serrano de Haro, A., La precisión del cuerpo. Análisis filosófico de la puntería, Madrid, Trotta, 2007, pp.88, 89.

12 Idem., p. 87.

13 Idem.

14 Sokolowski, R., Ibid., pp. 103, 104.

15 Ingarden, R., Ontology of the Work of Art. The Musical Work, The Picture, The Architectural Work, The Film. Ohio University press, 1989, p. 266. "En la obra arquitectónica es necesario distinguir entre dos estratos. Éstos son: 1) Los aspectos visuales en los que se muestra fenoménicamente la forma espacial de la obra arquitectónica; 2) La forma tridimensional de esta obra (la catedral, el teatro, etc.) que aparece en esos aspectos" [traducción mía]. (Versión original en alemán Untersuchungen zur Ontologie der Kunst, Max Niemeyer, 1961.)

16 lbid., p. 259. También, p. 262: "Al todo consistente en muros lo llamo, por mor de la brevedad, el "edificio".

17 Idem., p. 259.

18 lbid., p. 261.

19 Ibid., p. 270. "1) La forma espacial objetiva que lleva o puede llevar en sí misma varias cualidades de valor estético, y 2) una multiplicidad de esquemas de aspectos a través de los cuales la forma espacial se hace presente cuando un espectador, en su experiencia consciente, actualiza esos esquemas $y$, asi, los concretiza de tal manera que ellos también contienen ciertas cualidades de valor estético."

20 Idem.

21 Ibid., p. 303.

22 Ingarden, R., The Literary Work of Art. An Investigation on the Borderlines of Ontology, Logic and Theory of Literature. With an Appendix on the Functions of Language in the Theatre, Evanston, Northwestern University Press, 1973, §41, p. 262. (Versión original: Das literarische Kusntwerk: Eine Untersuchung aus dem Grenzgebiet der Ontologie, Logik und Literaturwissenschaft, Halle, 1931.) Hay traducción castellana: La obra de arte literaria. Bases ontológicas para una filosofía de la literatura, México, Taurus, 1998.

23 lbid., § 42, p. 265.

24 Hua. III, § 44, p. 100.

25 Valéry, P., Escritos sobre Leonardo da Vinci, Madrid, Visor, 1996, pp. 27, 28.

26 Valéry, P., Teoría poética y estética, "Poesía y pensamiento abstracto", Madrid, Visor, 1990, p. 85.

27 Ibid., p. 93: "Por el contrario, el poema no muere por haber vivido".

28 "minutos sin valor... percepciones sin porvenir". Valéry, P., Piezas sobre arte, "Degas Danza Dibujo", Madrid, Visor, 1999, p. 44.

29 Valéry, P., Ibid., "El infinito estético", p. 190.

30 Valéry, P., Teoría poética y estética, op. cit., p. 94.

31 Ingarden, R., Ontology of the Work of Art, op. cit., p. 303.
32 Habría que añadir a estos dos conceptos el de soberanía, correspondiente a la teoria deconstructiva (Derrida), según el cual la experiencia estética transgrede las otras experiencias, erigiéndose el arte como crítica de la razón. La soberanía presupone el concepto de autonomía. Ver Menke, Ch., La soberanía del arte. La experiencia estética según Adorno y Derrida, Madrid, Visor, 1997.

33 Ibid., p. 23.

34 lbid., p. 123

35 Valéry, P., Piezas sobre arte, op. cit., p. 60.

36 Menke, Ch., Op. cit., p. 129. Sobre esta subversión la deconstrucción erige el concepto de soberanía, rechazado frontalmente por la hermenéutica.

37 Gadamer, H.-G., Wahrheit und Methode. Grundzüge einer philosophischen Hermeneutik, Tübingen: J.C.B., Mohr (Paul Siebeck), 1965, p. 109.

\section{BIBLIOGRAFÍA}

Husserl, E. (1950): Ideen zu einer reinen Phänomenologie und phänomenologischen Philosophie. Erstes Buch, Husserliana Band III, Martinus Nijhoff.

- (1984): Logische Untersuchungen. Husserliana Band XIX, Martinus Nijhoff.

- (1954): Erfahrung und Urteil, Ed. L. Landgrebe, Hamburg, Claassen.

Gadamer, H.-G. (1965): Wahrheit und Methode. Grundzüge einer philosophischen Hermeneutik, Tübingen, J.C.B. Mohr (Paul Siebeck) (2. Auflage).

Ingarden, R. (1989): Ontology of the Work of Art. The Musical Work, The Picture, The Architectural Work, The Film, Ohio University Press.

- $\quad$ (1973): The Literary Work of Art. An Investigation on the Borderlines of Ontology, Logic and Theory of Literature. 
With an Appendix on the Functions of Language in the Theatre, Evanston, Northwestern University Press.

Menke, Ch. (1997): La soberanía del arte. La experiencia estética según Adorno y Derrida, Madrid, Visor.
Serrano de Haro, A. (2007): La precisión del cuerpo. Análisis filosófico de la puntería, Madrid, Trotta.

Sokolowski, R. (1974): Husserlian Meditations. How Words Present Things, Evanston, Northwestern University Press.
Valéry, P. (1996): Escritos sobre Leonardo da Vinci, Madrid, Visor.

- (1990): Teoría poética y estética, Madrid, Visor.

- (1999): Piezas sobre arte, Madrid, Visor. 